seit dem 1.4.2009 Vergütungsstrukturen im ambulanten vertragsärztlichen Bereich vor, die sich stärker an der Morbidität der Versicherten orientieren sollen. Ab dem 1.1.2011 ist deshalb vorgesehen, die bisherige verpflichtende Verwendung des ICD 10 zur Diagnosekodierung durch sog. ambulante Kodierrichtlinien (AKR) auszuweiten.

\section{MMW Kommentar}

Während für den fachärztlichen und insbesondere auch den stationären Bereich die Orientierung der Honorierung an eine Morbiditätsentwicklung sinnvoll erscheint, wird die Ausrichtung der hausärz- tlichen Vergütung an solchen Kodierrichtlinien und einem daraus resultierenden Morbi-RSA ad absurdum geführt.

Wie eine Auswertung der Ergebnisse der Sächsischen Epidemiologischen Studie in der Allgemeinmedizin (SESAM-2) kürzlich aufgezeigt hat, gibt es nämlich extreme Unterschiede bei der Diagnosekodierung in den einzelnen Kapiteln und insbesondere bei drei- bzw. vierstelliger Verschlüsselung war kaum noch ein Konsens unter den Ratern zu beobachten. Die fortwährende Erweiterung des Diagnosenschlüssels, und die Verpflichtung zur endständigen Verschlüsselung sind hier deshalb äußerst kritisch zu betrachten.
Allein schon die Reliabilität der ICD-10-Kodierung kann in der Allgemeinmedizin als nicht ausreichend angesehen werden. Ziel einer an die Besonderheiten der Hausarztmedizin angepassten Qualitätssicherung und Diagnosekodierung muss ein für die hausärztliche Tätigkeit eigenständiges Kodiersystem sein, das der eigentlichen Zielsetzung der Hausarztmedizin, dem Verhindern von Erkrankungen, Rechnung trägt. Dies erfordert im zweiten Schritt nicht nur eine Abtrennung der hausärztlichen Vergütung von der morbiditätsbedingten Gesamtvergütung, sondern auch eine vom Morbi-RSA unabhängige finanzielle Ausstattung.

\title{
Heilmittelausgaben 2009 stark angestiegen: Vorsicht Regress!
}

Die GKV-Heilmittelfrühinformationen für das Jahr 2009 stehen jetzt zur Verfügung. Diese Daten stammen von den Krankenkassenverbänden und stellen später die Basis für Wirtschaftlichkeits- oder Richtgrößenprüfungen dar. Da sie die Grundlage für Regresse sein können, sollte man schon bei der Verordnung Angriffspunkte vermeiden.

\section{MMW Kommentar}

Die Prüfgremien urteilen in der Regel nach Kriterien, die im Vorfeld beachtet werden können. So zählen Massagen und Wärmeanwendungen zu den sog. passiven Behandlungen. Diese gelten zwar als wirksam, die Therapieerfolge halten aber nur begrenzt an, so dass aktive Behandlungen wie z.B. die Krankengymnastik bei der Verordnung favorisiert werden sollten.

Der Heilmittelkatalog geht davon aus, dass eine Erkrankung im Regelfall entsprechend der Heilmittelrichtlinie mit einer bestimmten Anzahl an Therapieeinheiten ausreichend behandelt ist. Ist eine Behandlung darüber hinaus erforderlich, kann sie mit einer medizinischen Begründung und einer prognostischen Einschätzung zum Krankheitsverlauf als Verordnung außerhalb des Regelfalles vorgenommen werden. Je differenzierter die Verordnungen dokumentiert sind, umso leichter kann die Prüfungsstelle die Notwendigkeit der Therapie im Prüfungsfall nachvollziehen. Ein
Hausbesuch verteuert die Behandlungskosten. Er muss deshalb medizinisch und nicht organisatorisch begründet sein und die Verordnung von physikalischer Therapie kann nicht die mobilisierende Pflege ersetzen, die von Pflegeeinrichtungen zu leisten ist. Eine Therapie kann auch nicht alleine deshalb veranlasst werden, weil der Patient seinen Urlaub in einem „KurOrt" verbringt.

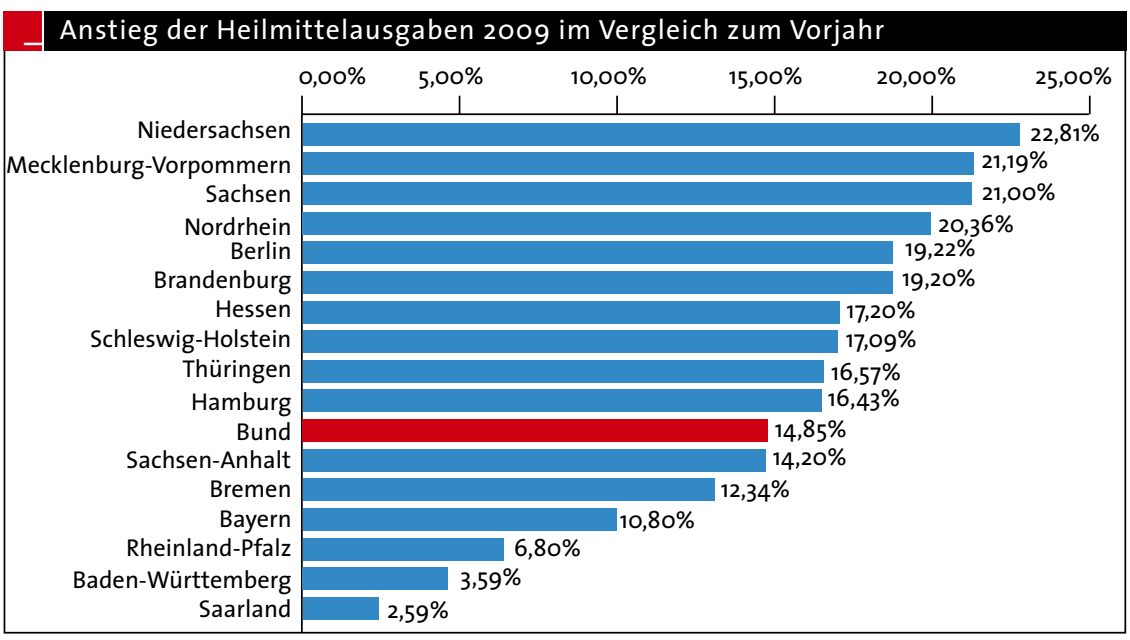

